\title{
LP-SASAKIAN MANIFOLDS EQUIPPED WITH ZAMKOVOY CONNECTION AND CONHARMONIC CURVATURE TENSOR
}

\author{
Abhijit Mandal ${ }^{1}$, Ashoke Das ${ }^{2}$ \\ ${ }^{1}$ Raiganj Surendranath Mahavidyalaya, Raiganj, \\ Uttar Dinajpur, West Bengal, India-733134. \\ Email: abhijit4791@gmail.com \\ ${ }^{2}$ Raiganj University, Raiganj, Uttar Dinajpur, \\ West Bengal, India-733134. \\ Email: ashoke.avik@gmail.com
}

\begin{abstract}
The paper concerns with some results on conharmonically flat, quasiconharmonically flat and $\phi$-conharmonically flat LP-Sasakian manifolds with respect to Zamkovoy connection. Also, it contains study of generalized conharmonic $\phi$-recurrent LP-Sasakian manifolds with respect to Zamkovoy connection. Moreover, the paper deals with LP-Sasakian manifolds satisfying $\mathcal{K}^{*}(\xi, U) \cdot R^{*}=0$, where $\mathcal{K}^{*}$ denotes conharmonic curvature tensor and $R^{*}$ denotes Riemannian curvature tensor with respect to Zamkovoy connection, respectively.

Key words and Phrases: LP-Sasakian manifold, Zamkovoy connection, Conharmonic curvature tensor
\end{abstract}

\section{INTRODUCTION}

In 1989, K. Matsumoto [13] first introduced the notion of Lorentzian paraSasakian manifolds (briefly, LP-Sasakian manifolds). Also, in 1992, I. Mihai and R. Rosca [14] introduced independently the notion of Lorentzian para-Sasakian manifolds in classical analysis. The generalized recurrent manifolds was introduced by Dubey [8] and it was studied by De and Guha et al. [6]. In this context, $\phi$-recurrent LP-Sasakian manifold was first studied by A. A. Shaikh, D. G. Prakasha and Helaluddin Ahmad [15]. On the other hand, $\phi$-conharmonically flat LP-Sasakian manifold was introduced by A. Taleshian [16]. Apart from these, the properties of LP-Sasakian manifolds were studied by several authors, namely U. C. De [7], C. Ozgur [17] and many others.

2020 Mathematics Subject Classification: 53C15, 53C50 Received: 02-10-2020, accepted: 06-04-2021. 
In 2008, a new non-metric canonical connection on para contact manifold was introduced by S. Zamkovoy [18]. This connection named as Zamkovoy connection was further studied in Sasakian manifolds, LP-Sasakian manifolds and paraKenmotsu manifolds by several researcher et al. ([3], [1], [2], [10], [11], [12], [5]). Zamkovoy connection $\nabla^{*}$ for an $n$-dimensional almost contact metric manifold [4] $M$ equipped with an almost contact metric structure $(\phi, \xi, \eta, g)$ consisting of a $(1,1)$ tensor field $\phi$, a vector field $\xi$, a 1 -form $\eta$ and a Riemannian metric $g$ is given by

$$
\nabla_{X}^{*} Y=\nabla_{X} Y+\left(\nabla_{X} \eta\right)(Y) \xi-\eta(Y) \nabla_{X} \xi+\eta(X) \phi Y,
$$

for all $X, Y \in \chi(M)$, where $\nabla$ is the Levi-Civita connection and $\chi(M)$ is the set of all vector fields on $M$.

In 1957, Y. Ishii [9] first studied the notion of a conharmonic curvature tensor. A rank three tensor $\mathcal{K}$, that remains invariant under conharmonic transformation for an $n$-dimensional Riemannian manifold $M$ is given by

$$
\begin{aligned}
\mathcal{K}(X, Y) Z= & R(X, Y) Z-\frac{1}{n-2}[S(Y, Z) X-S(X, Z) Y] \\
& -\frac{1}{n-2}[g(Y, Z) Q X-g(X, Z) Q Y]
\end{aligned}
$$

for all $X, Y, Z \in \chi(M)$, where $\chi(M)$ is the set of all vector fields of the manifold $M$ and $R$ denotes the Riemannian curvature tensor of type $(1,3), S$ denotes the Ricci tensor of type $(0,2), Q$ is the Ricci operator.

The conharmonic curvature tensor $\left(\mathcal{K}^{*}\right)$ with respect to Zamkovoy connection is given by

$$
\begin{aligned}
\mathcal{K}^{*}(X, Y) Z= & R^{*}(X, Y) Z-\frac{1}{n-2}\left[S^{*}(Y, Z) X-S^{*}(X, Z) Y\right] \\
& -\frac{1}{n-2}\left[g(Y, Z) Q^{*} X-g(X, Z) Q^{*} Y\right],
\end{aligned}
$$

for all $X, Y, Z \in \chi(M)$, where $R^{*}, S^{*}$ and $Q^{*}$ are Riemannian curvature tensor, Ricci tensor and Ricci operator with respect to Zamkovoy connection, respectively.

Definition 1.1. An n-dimensional LP-Sasakian manifold $M$ is said to be generalized $\eta$-Einstein manifold if the Ricci tensor of type $(0,2)$ is of the form

$$
S(Y, Z)=k_{1} g(Y, Z)+k_{2} \eta(Y) \eta(Z)+k_{3} \omega(Y, Z),
$$

for all $Y, Z \in \chi(M)$, where $k_{1}, k_{2}$ and $k_{3}$ are scalars and $\omega$ is a 2 -form.

Definition 1.2. An n-dimensional LP-Sasakian manifold $M$ is said to be conharmonically flat with respect to Zamkovoy connection if $\mathcal{K}^{*}(X, Y) Z=0$, for all $X, Y, Z \in \chi(M)$.

Definition 1.3. An n-dimensional LP-Sasakian manifold $M$ is said to be $\xi-$ conharmonically flat with respect to Zamkovoy connection if $\mathcal{K}(X, Y) \xi=0$, for all $X, Y, Z \in \chi(M)$. 
Definition 1.4. An n-dimensional LP-Sasakian manifold $M$ is said to be generalized conharmonic $\phi$-recurrent with respect to Zamkovoy connection if

$$
\begin{aligned}
\phi^{2}\left(\nabla_{W}^{*} \mathcal{K}^{*}\right)(X, Y) Z= & A(W) K(X, Y) Z \\
& +B(W)[g(Y, Z) X-g(X, Z) Y],
\end{aligned}
$$

for all $X, Y, Z, W \in \chi(M)$, where $A$ and $B$ are 1 -forms and $B$ is non vanishing such that $A(W)=g\left(W, \rho_{1}\right), B(W)=g\left(W, \rho_{2}\right)$ and $\rho_{1}, \rho_{2}$ are vector fields associated with 1 -forms $A$ and $B$, respectively.

This paper is structured as follows:

After introduction, a short description of LP-Sasakian manifold has been given in section (2). In section (3), we have obtained Riemannian curvature tensor $R^{*}$, Ricci tensor $S^{*}$, scalar curvature $r^{*}$ with respect to Zamkovoy connection in LP-Sasakian manifold. Section (4) contains conharmonically flat and $\xi$-conharmonically flat LP-Sasakian manifolds with respect to Zamkovoy connection. In section (5), we have discussed quasi-conharmonically flat LP-Sasakian manifold with respect to Zamkovoy connection. Section (6) contains $\phi-$ conharmonically flat LP-Sasakian manifold with respect to $\nabla^{*}$. Section (7) concerns with a generalized conharmonic $\phi$-recurrent LP-Sasakian manifold with respect to $\nabla^{*}$. In section (8), we have discussed an LP-Sasakian manifold satisfying $\mathcal{K}^{*}(\xi, U) \cdot R^{*}=0$.

\section{Preliminaries}

An $n$-dimensional differentiable manifold is called an LP-Sasakian manifold if it admits a $(1,1)$ tensor field $\phi$, a vector field $\xi$, a 1 -form $\eta$ and a Lorentzian metric $g$ which satisfies:

$$
\begin{aligned}
\phi^{2} Y & =Y+\eta(Y) \xi, \eta(\xi)=-1, \eta(\phi X)=0, \phi \xi=0, \\
g(\phi X, \phi Y) & =g(X, Y)+\eta(X) \eta(Y), \\
g(X, \phi Y) & =g(\phi X, Y), \eta(Y)=g(Y, \xi), \\
\nabla_{X} \xi & =\phi X, \quad g(X, \xi)=\eta(X), \\
\left(\nabla_{X} \phi\right) Y & =g(X, Y) \xi+\eta(Y) X+2 \eta(X) \eta(Y) \xi,
\end{aligned}
$$

for all $X, Y \in \chi(M)$, where $\nabla$ denotes the operator of covariant differentiation with respect to the Lorentzian metric $g$.

Let us introduced a symmetric $(0,2)$ tensor field $\omega$ such that

$$
\omega(X, Y)=g(X, \phi Y) .
$$

Also, since the vector field $\eta$ is closed in LP-Sasakian manifold $M$, we have

$$
\left(\nabla_{X} \eta\right) Y=\omega(X, Y), \omega(X, \xi)=0,
$$

for all $X, Y \in \chi(M)$. 
In LP-Sasakian manifold the following relations also hold:

$$
\begin{aligned}
\eta(R(X, Y) Z) & =g(Y, Z) \eta(X)-g(X, Z) \eta(Y), \\
R(X, Y) \xi & =\eta(Y) X-\eta(X) Y, \\
R(\xi, Y) Z & =g(Y, Z) \xi-\eta(Z) Y, \\
R(\xi, Y) \xi & =\eta(Y) \xi+Y, \\
S(X, \xi) & =(n-1) \eta(X), \\
S(\phi X, \phi Y) & =S(X, Y)+(n-1) \eta(X) \eta(Y), \\
Q \xi=(n-1) \xi, Q \phi=\phi Q, S & (X, Y)=g(Q X, Y), S^{2}(X, Y)=S(Q X, Y) .
\end{aligned}
$$

Lemma 2.1. The relation between Zamkovoy connection and Levi-Civita connection in an LP-Sasakian manifold is given by

$$
\nabla_{X}^{*} Y=\nabla_{X} Y+g(X, \phi Y) \xi-\eta(Y) \phi X+\eta(X) \phi Y,
$$

where the torsion tensor of Zamkovoy connection is

$$
T^{*}(X, Y)=2[\eta(X) \phi Y-\eta(Y) \phi X] .
$$

Proof. In view of (1) and (11), we have

$$
\left(\nabla_{X}^{*} g\right)(Y, Z)=-2 g(Y, \phi Z) \eta(X) .
$$

Suppose that the Zamkovoy connection $\nabla^{*}$ defined on an $n$ - dimensional LPSasakian manifold $M$ is connected with the Levi-Civita connection $\nabla$ by the relation

$$
\nabla_{X}^{*} Y=\nabla_{X} Y+P(X, Y),
$$

where $P(X, Y)$ is a tensor field of type $(1,1)$. Then by definition of torsion tensor, we have

$$
T^{*}(X, Y)=P(X, Y)-P(Y, X) .
$$

Zamkovoy connection is a non-metric connection and hence from (22), we get

$$
\begin{aligned}
g(P(X, Y), Z)+g(P(X, Z), Y) & =2 g(Y, \phi Z) \eta(X), \\
g(P(Y, X), Z)+g(P(Y, Z), X) & =2 g(X, \phi Z) \eta(Y), \\
g(P(Z, X), Y)+g(P(Z, Y), X) & =2 g(X, \phi Y) \eta(Z) .
\end{aligned}
$$

In view of (24), (25), (26) and (23), we have

$$
\begin{aligned}
& g\left(T^{*}(X, Y), Z\right)+g\left(T^{*}(Z, X), Y\right)+g\left(T^{*}(Z, Y), X\right) \\
= & g(P(X, Y), Z)-g(P(Y, X), Z)+g(P(Z, X), Y) \\
& -g(P(X, Z), Y)+g(P(Z, Y), X)-g(P(Y, Z), X) \\
= & 2 g(P(X, Y), Z)-2 g(Y, \phi Z) \eta(X) \\
& -2 g(X, \phi Z) \eta(Y)+2 g(X, \phi Y) \eta(Z) .
\end{aligned}
$$

Setting

$$
\begin{aligned}
& g\left(T^{*}(Z, X), Y\right)=g(\bar{T}(X, Y), Z), \\
& g\left(T^{*}(Z, Y), X\right)=g(\bar{T}(Y, X), Z),
\end{aligned}
$$


in (27), we have

$$
\begin{aligned}
& g\left(T^{*}(X, Y), Z\right)+g(\bar{T}(X, Y), Z)+g(\bar{T}(Y, X), Z) \\
= & 2 g(P(X, Y), Z)-2 g(Y, \phi Z) \eta(X) \\
& -2 g(X, \phi Z) \eta(Y)+2 g(X, \phi Y) \eta(Z)
\end{aligned}
$$

which implies that

$$
\begin{aligned}
P(X, Y)= & \frac{1}{2}\left[T^{*}(X, Y)+\bar{T}(X, Y)+\bar{T}(Y, X)\right] \\
& +\eta(X) \phi Y+\eta(Y) \phi X-g(X, \phi Y) \xi .
\end{aligned}
$$

In reference to $(20),(28)$ and $(29)$, we have

$$
\begin{aligned}
& \bar{T}(X, Y)=2 g(X, \phi Y) \xi-2 \eta(X) \phi Y, \\
& \bar{T}(Y, X)=2 g(X, \phi Y) \xi-2 \eta(Y) \phi X .
\end{aligned}
$$

Using (20), (32) and (33) in (31), we obtain

$$
P(X, Y)=g(X, \phi Y) \xi-\eta(Y) \phi X+\eta(X) \phi Y .
$$

In reference to (22) and (34), we can easily bring out the equation (19).

From the equation (19), it is obvious that

$$
\nabla_{X}^{*} \xi=2 \phi X .
$$

Proposition 2.2. The Zamkovoy connection on an n-dimensional LP-Sasakian manifold is a non-metric linear connection with torsion tensor given by equation (20).

\section{Some Properties of LP-SASAKIAN MANIFOLD With RESPECT to ZAMKOVOY CONNECTION}

Let $R^{*}$ be the Riemannian curvature tensor with respect to Zamkovoy connection and it be defined as

$$
R^{*}(X, Y) Z=\nabla_{X}^{*} \nabla_{Y}^{*} Z-\nabla_{Y}^{*} \nabla_{X}^{*} Z-\nabla_{[X, Y]}^{*} Z .
$$

Using (5), (8), (9) and (19) in (36), we get the Riemannian curvature $R^{*}$ with respect to Zamkovoy connection as

$$
\begin{aligned}
R^{*}(X, Y) Z= & R(X, Y) Z+3 g(X, Z) \eta(Y) \xi \\
& -3 g(Y, Z) \eta(X) \xi+3 g(Y, \phi Z) \phi X-3 g(X, \phi Z) \phi Y \\
& -\eta(X) \eta(Z) Y+\eta(Y) \eta(Z) X .
\end{aligned}
$$

Consequently, one can easily bring out the followings:

$$
\begin{aligned}
S^{*}(Y, Z) & =S(Y, Z)+(n-1) \eta(Y) \eta(Z)+3 \psi g(Y, \phi Z), \\
S^{*}(\xi, Z) & =S^{*}(Z, \xi)=0, \\
Q^{*} Y & =Q Y+(n-1) \eta(Y) \xi+3 \psi \phi Y, \\
Q^{*} \xi & =0, \\
r^{*} & =r-n+1+3 \psi^{2},
\end{aligned}
$$




$$
\begin{aligned}
R^{*}(X, Y) \xi & =0 \\
R^{*}(\xi, Y) Z & =4 g(\phi Y, \phi Z) \xi, \\
R^{*}(X, \xi) Z & =-4 g(\phi X, \phi Z) \xi
\end{aligned}
$$

for all $X, Y, Z \in \chi(M)$, where $\psi=\operatorname{trace}(\phi)$.

Proposition 3.1. Let $M$ be an n-dimensional LP-Sasakian manifold admitting Zamkovoy connection $\nabla^{*}$, then

(i) The curvature tensor $R^{*}$ of $\nabla^{*}$ is given by (37),

(ii) The Ricci tensor $S^{*}$ of $\nabla^{*}$ is given by (38),

(iii) The scalar curvature $r^{*}$ of $\nabla^{*}$ is given by (42),

(iv) The Ricci tensor $S^{*}$ of $\nabla^{*}$ is symmetric,

(v) $R^{*}$ satisfies: $R^{*}(X, Y) Z+R^{*}(Y, Z) X+R^{*}(Z, X) Y=0$.

\section{Conharmonically flat and $\xi$-CONHARmonically flat LP-Sasakian} MANIFOLDS WITH RESPECT TO ZAMKOVOY CONNECTION

Theorem 4.1. If an $n$-dimensional LP-Sasakian manifold $M(n>2)$ is conharmonically flat with respect to Zamkovoy connection, then the scalar curvature is given by $r=n-1-3 \psi^{2}$.

Proof. In view of (2) and (3), we have

$$
\begin{aligned}
& \mathcal{K}^{*}(X, Y) Z \\
= & \mathcal{K}(X, Y) Z+3 g(X, Z) \eta(Y) \xi-3 g(Y, Z) \eta(X) \xi \\
& +3 g(Y, \phi Z) \phi X-3 g(X, \phi Z) \phi Y-\eta(X) \eta(Z) Y+\eta(Y) \eta(Z) X \\
& -\frac{n-1}{n-2}[g(Y, Z) \eta(X) \xi-g(X, Z) \eta(Y) \xi] \\
& -\frac{3 \psi}{n-2}[g(Y, Z) \phi X-g(X, Z) \phi Y] \\
& -\frac{n-1}{n-2}[\eta(Y) X-\eta(X) Y] \eta(Z) \\
& -\frac{3 \psi}{n-2}[g(Y, \phi Z) X-3 \psi g(X, \phi Z) Y] .
\end{aligned}
$$

Let us consider an LP-Sasakian manifold $M$ which is conharmonically flat with respect to Zamkovoy connection, then from (3), we have

$$
\begin{aligned}
R^{*}(X, Y) Z= & \frac{1}{n-2}\left[S^{*}(Y, Z) X-S^{*}(X, Z) Y\right] \\
& +\frac{1}{n-2}\left[g(Y, Z) Q^{*} X+g(X, Z) Q^{*} Y\right] .
\end{aligned}
$$


Taking inner product of (47) with a vector field $V$, we get

$$
\begin{aligned}
& g\left(R^{*}(X, Y) Z, V\right) \\
= & \frac{1}{n-2}\left[S^{*}(Y, Z) g(X, V)-S^{*}(X, Z) g(Y, V)\right] \\
& +\frac{1}{n-2}\left[g(Y, Z) S^{*}(X, V)-g(X, Z) S^{*}(Y, V)\right] .
\end{aligned}
$$

Taking an orthonormal frame field of $M$ and contracting (48) over $X$ and $V$, we obtain

$$
r=n-1-3 \psi^{2}
$$

This gives the theorem.

Corollary 4.2. If an LP-Sasakian manifold is conharmonically flat with respect to Zamkovoy connection, then its scalar curvature is constant, provided that trace $(\phi)=$ 0 .

Theorem 4.3. An $n$-dimensional LP-Sasakian manifold $(n>2)$ is $\xi-$ conharmonically flat with respect to Zamkovoy connection if and only if it is so with respect to Levi-Civita connection, provided that the vector fields are horizontal vector fields.

Proof. Setting $Z=\xi$ in (46), we have

$$
\begin{aligned}
& \mathcal{K}^{*}(X, Y) \xi \\
= & \mathcal{K}(X, Y) \xi+\frac{1}{n-2}[\eta(Y) X-\eta(X) Y] \\
& -\frac{3 \psi}{n-2}[\eta(Y) \phi X-\eta(X) \phi Y] \\
= & \mathcal{K}(X, Y) \xi, \quad \text { if } X, Y \text { are horizontal vector fields on } M .
\end{aligned}
$$

This gives the theorem.

Theorem 4.4. If an $n$-dimensional LP-Sasakian manifold $(n>2)$ is $\xi-$ conharmonically flat with respect to Zamkovoy connection, then its scalar curvature with respect to Zamkovoy connection vanishes.

Proof. Setting $Z=\xi$ in (3), we have

$$
\mathcal{K}^{*}(X, Y) \xi=\frac{1}{n-2}\left[\eta(Y) Q^{*} X-\eta(X) Q^{*} Y\right]
$$

If $M$ is $\xi$-conharmonically flat with respect to Zamkovoy connection, then it follows from (50) that

$$
0=\eta(Y) Q^{*} X-\eta(X) Q^{*} Y
$$

Taking inner product of (51) with a vector field $V$, we obtain

$$
0=\eta(Y) S^{*}(X, V)-\eta(X) S^{*}(Y, V) .
$$

Setting $Z=\xi$ in $(52)$

$$
S^{*}(X, V)=0 \text {. }
$$


Taking an orthonormal frame field of $M$ and contracting (53) over $X$ and $V$, we get

$$
r^{*}=0
$$

This gives the theorem.

5. QUASI-CONHARMONICALLY FLAT LP-SASAKIAN MANIFOLD WITH RESPECT TO ZAMKOVOY CONNECTION.

Theorem 5.1. If an $n$-dimensional LP-Sasakian manifold $M(n>2)$ is quasiconharmonically flat with respect to Zamkovoy connection, then its scalar curvature with respect to Zamkovoy connection vanishes.

Proof. Let us consider an LP-Sasakian manifold $M$ which is quasi-conharmonically flat with respect to Zamkovoy connection, i.e.,

$$
g\left(\mathcal{K}^{*}(\phi X, Y) Z, \phi V\right)=0,
$$

for all $X, Y, Z, V \in \chi(M)$.

Then, in view of (3), we have

$$
\begin{aligned}
& g\left(R^{*}(\phi X, Y) Z, \phi V\right) \\
= & \frac{1}{n-2}\left[S^{*}(Y, Z) g(\phi X, \phi V)-S^{*}(\phi X, Z) g(Y, \phi V)\right] \\
& +\frac{1}{n-2}\left[g(Y, Z) S^{*}(\phi X, \phi V)-g(\phi X, Z) S^{*}(Y, \phi V)\right] .
\end{aligned}
$$

Let $\left\{e_{i}\right\}(1 \leq i \leq n)$ be an orthonormal basis of the tangent space at any point of the manifold $M$. Setting $Y=Z=e_{i}$ in the equation (55) and taking summation over $i(1 \leq i \leq n)$, we get

$$
\begin{aligned}
& \sum_{i=1}^{n} g\left(R^{*}\left(\phi X, e_{i}\right) e_{i}, \phi V\right) \\
= & \frac{1}{n-2}\left[\sum_{i=1}^{n} S^{*}\left(e_{i}, e_{i}\right) g(\phi X, \phi V)-\sum_{i=1}^{n} S^{*}\left(\phi X, e_{i}\right) g\left(e_{i}, \phi V\right)\right] \\
& +\frac{1}{n-2}\left[\sum_{i=1}^{n} g\left(e_{i}, e_{i}\right) S^{*}(\phi X, \phi V)-\sum_{i=1}^{n} g\left(\phi X, e_{i}\right) S^{*}\left(e_{i}, \phi V\right)\right] .
\end{aligned}
$$

It can be easily seen that

$$
\begin{aligned}
\sum_{i=1}^{n} g\left(e_{i}, e_{i}\right) & =n, \\
\sum_{i=1}^{n} S^{*}\left(\phi X, e_{i}\right) g\left(e_{i}, \phi V\right) & =S^{*}(\phi X, \phi V), \\
\sum_{i=1}^{n} S^{*}\left(e_{i}, e_{i}\right) & =r^{*} .
\end{aligned}
$$


Using (57), (58) and (59) in (56), we get

$$
r^{*}=0
$$

This gives the theorem.

6. $\phi$-CONHARMONICALLY FLAT LP-SASAKIAN MANIFOLD WITH RESPECT TO ZAMKOVOY CONNECTION

Theorem 6.1. If an $n$-dimensional LP-Sasakian manifold $M(n>2)$ is $\phi$-conharmonically flat with respect to Zamkovoy connection, then $M$ is a generalized $\eta$-Einstein manifold.

Proof. Let us consider an LP-Sasakian manifold $M$ which is $\phi$-conharmonically flat with respect to Zamkovoy connection, i.e.,

$$
g\left(\mathcal{K}^{*}(\phi X, \phi Y) \phi Z, \phi V\right)=0
$$

for all $X, Y, Z, V \in \chi(M)$

Then in view of (3), we have

$$
\begin{aligned}
& g\left(R^{*}(\phi X, \phi Y) \phi Z, \phi V\right) \\
= & \frac{1}{n-2}\left[S^{*}(\phi Y, \phi Z) g(\phi X, \phi V)-S^{*}(\phi X, \phi Z) g(\phi Y, \phi V)\right] \\
& +\frac{1}{n-2}\left[g(\phi Y, \phi Z) S^{*}(\phi X, \phi V)-g(\phi X, \phi Z) S^{*}(\phi Y, \phi V)\right] .
\end{aligned}
$$

Let $\left\{e_{i}, \xi\right\}(1 \leq i \leq n-1)$ be a local orthonormal basis of the tangent space at any point of the manifold $M$. Using the fact that $\left\{\phi e_{i}, \xi\right\}(1 \leq i \leq n-1)$ is also a local orthonormal basis of the tangent space and setting $Y=Z=e_{i}$ and taking summation over $i(1 \leq i \leq n-1)$ it follows from (61) that

$$
\begin{aligned}
& \sum_{i=1}^{n-1} R^{*}\left(\phi X, \phi e_{i}, \phi e_{i}, \phi V\right) \\
= & \frac{1}{n-2}\left[\sum_{i=1}^{n-1} S^{*}\left(\phi e_{i}, \phi e_{i}\right) g(\phi X, \phi V)-\sum_{i=1}^{n-1} S^{*}\left(\phi X, \phi e_{i}\right) g\left(\phi e_{i}, \phi V\right)\right] \\
& +\frac{1}{n-2}\left[\sum_{i=1}^{n-1} g\left(\phi e_{i}, \phi e_{i}\right) S^{*}(\phi X, \phi V)-\sum_{i=1}^{n-1} g\left(\phi X, \phi e_{i}\right) S^{*}\left(\phi e_{i}, \phi V\right)\right]
\end{aligned}
$$


It can be easily seen that

$$
\begin{aligned}
\sum_{i=1}^{n-1} g\left(\phi e_{i}, \phi e_{i}\right) & =n-1, \\
\sum_{i=1}^{n-1} S^{*}\left(\phi X, \phi e_{i}\right) g\left(\phi e_{i}, \phi V\right) & =S^{*}(\phi X, \phi V), \\
\sum_{i=1}^{n-1} S^{*}\left(\phi e_{i}, \phi e_{i}\right) & =r^{*}
\end{aligned}
$$

Using (63), (64) and (65) in (62), we have

$$
\begin{aligned}
& S(X, V)=\left(r-n+1+3 \psi^{2}\right) g(X, V) \\
& +\left(r-2 n+2+3 \psi^{2}\right) \eta(X) \eta(V)-3 \psi \omega(X, V),
\end{aligned}
$$

where $\omega(X, V)=g(X, \phi V)$ and $\psi=\operatorname{trace}(\phi)$.

Therefore $M$ is a generalized $\eta$-Einstein manifold.

7. Generalized CONHARMOnic $\phi$-RECURREnT LP-SASAKIAN MANifold With RESPECT TO ZAMKOVOY CONNECTION

Theorem 7.1. If an $n$-dimensional LP-Sasakian manifold $M(n>2)$ is generalized conharmonic $\phi$-recurrent with respect to Zamkovoy connection, then 1 -forms $A$ and $B$ are related as $B(W)=\left[\frac{r-n+1+3 \psi^{2}}{(n-2)(n-1)}\right] A(W)$, where $W$ is an arbitrary vector field on $M$ and $\psi=\operatorname{trace}(\phi)$.

Proof. Let $M$ be a generalized conharmonic $\phi$-recurrent LP-Sasakian manifold with respect to Zamkovoy connection, then

$$
\begin{aligned}
& \phi^{2}\left(\nabla_{W}^{*} \mathcal{K}^{*}\right)(X, Y) Z \\
= & A(W) \mathcal{K}^{*}(X, Y) Z+B(W)[g(Y, Z) X-g(X, Z) Y],
\end{aligned}
$$

where the 1 -forms are given by $A(W)=g\left(W, \rho_{1}\right), B(W)=g\left(W, \rho_{2}\right), B(W) \neq$ 0 and $\rho_{1}, \rho_{2}$ are vector fields associated with 1 -forms $A$ and $B$, respectively.

Using (5) in (67), we have

$$
\begin{aligned}
& \left(\nabla_{W}^{*} \mathcal{K}^{*}\right)(X, Y) Z \\
= & -\eta\left(\left(\nabla_{W}^{*} \mathcal{K}^{*}\right)(X, Y) Z\right) \xi A(W) \mathcal{K}^{*}(X, Y) Z \\
& +B(W)[g(Y, Z) X-g(X, Z) Y] .
\end{aligned}
$$

The inner product of the equation (68) with vector field $V$ gives

$$
\begin{aligned}
& g\left(\left(\nabla_{W}^{*} \mathcal{K}^{*}\right)(X, Y) Z, V\right) \\
=\quad & -\eta\left(\left(\nabla_{W}^{*} \mathcal{K}^{*}\right)(X, Y) Z\right) \eta(V)+A(W) g\left(\mathcal{K}^{*}(X, Y) Z, V\right) \\
+ & B(W)[g(Y, Z) g(X, V)-g(X, Z) g(Y, V)] .
\end{aligned}
$$


In view of (3), it is easily seen that

$$
\begin{aligned}
& g\left(\left(\nabla_{W}^{*} \mathcal{K}^{*}\right)(X, Y) Z, V\right) \\
= & g\left(\left(\nabla_{W}^{*} R^{*}\right)(X, Y) Z, V\right) \\
- & \frac{1}{n-2}\left[\left(\nabla_{W}^{*} S^{*}\right)(Y, Z) g(X, V)-\left(\nabla_{W}^{*} S^{*}\right)(X, Z) g(Y, V)\right] \\
- & \frac{1}{n-2}\left[g(Y, Z)\left(\nabla_{W}^{*} S^{*}\right)(X, V)-g(X, Z)\left(\nabla_{W}^{*} S^{*}\right)(Y, V)\right], \\
= & g\left(\left(\nabla_{W}^{*} R^{*}\right)(X, Y) Z, \xi\right) \\
& -\frac{1}{n-2}\left[\left(\nabla_{W}^{*} S^{*}\right)(Y, Z) \eta(X)-\left(\nabla_{W}^{*} S^{*}\right)(X, Z) \eta(Y)\right], \\
& \\
& \quad g\left(\mathcal{K}^{*}(X, Y) Z, V\right) \\
= & g\left(R^{*}(X, Y) Z, V\right) \\
& -\frac{1}{n-2}\left[S^{*}(Y, Z) g(X, V)-S^{*}(X, Z) g(Y, V)\right] \\
& -\frac{1}{n-2}\left[g(Y, Z) S^{*}(X, V)-g(X, Z) S^{*}(Y, V)\right] .
\end{aligned}
$$

Using (70), (71) and (72) in (69), we get

$$
\begin{aligned}
& g\left(\left(\nabla_{W}^{*} R^{*}\right)(X, Y) Z, V\right) \\
= & \frac{1}{n-2}\left[\left(\nabla_{W}^{*} S^{*}\right)(Y, Z) g(X, V)-\left(\nabla_{W}^{*} S^{*}\right)(X, Z) g(Y, V)\right] \\
& +\frac{1}{n-2}\left[g(Y, Z)\left(\nabla_{W}^{*} S^{*}\right)(X, V)-g(X, Z)\left(\nabla_{W}^{*} S^{*}\right)(Y, V)\right] \\
& +\frac{1}{n-2}\left[\left(\nabla_{W}^{*} S^{*}\right)(Y, Z) \eta(X)-\left(\nabla_{W}^{*} S^{*}\right)(X, Z) \eta(Y)\right] \eta(V) \\
& +g\left(R^{*}(X, Y) Z, V\right) A(W)-g\left(\left(\nabla_{W}^{*} R^{*}\right)(X, Y) Z, \xi\right) \eta(V) \\
& -\frac{1}{n-2}\left[S^{*}(Y, Z) g(X, V)-S^{*}(X, Z) g(Y, V)\right] A(W) \\
& -\frac{1}{n-2}\left[g(Y, Z) S^{*}(X, V)-g(X, Z) S^{*}(Y, V)\right] A(W) \\
& +[g(Y, Z) g(X, V)-g(X, Z) g(Y, V)] B(W) .
\end{aligned}
$$


Taking an orthonormal frame field of $M$ and contracting (73) over $Y$ and $Z$, we get

$$
\begin{aligned}
& \left(\nabla_{W}^{*} S^{*}\right)(X, V) \\
= & \frac{1}{n-2}\left[\nabla_{W}^{*} r^{*} g(X, V)-\left(\nabla_{W}^{*} S^{*}\right)(X, V)\right] \\
& +\frac{1}{n-2}\left[n\left(\nabla_{W}^{*} S^{*}\right)(X, V)-\left(\nabla_{W}^{*} S^{*}\right)(X, V)\right]-g\left(\nabla_{W}^{*} S^{*}\right)(X, \xi) \eta(V) \\
& +\frac{1}{n-2}\left[\nabla_{W}^{*} r^{*} \eta(X) \eta(V)-\left(\nabla_{W}^{*} S^{*}\right)(X, \xi) \eta(V)\right] \\
& +\left(\nabla_{W}^{*} S^{*}\right)(X, V) A(W)-\frac{1}{n-2}\left[r^{*} g(X, V)-S^{*}(X, V)\right] A(W) \\
& -\frac{n-1}{n-2} S^{*}(X, V) A(W)+(n-1) g(X, V) B(W) .
\end{aligned}
$$

Setting $V=\xi$ in $(74)$

$$
B(W)=\left[\frac{r-n+1+3 \psi^{2}}{(n-2)(n-1)}\right] A(W) .
$$

This gives the theorem.

\section{LP-SASAKIAN MANIFOLD SATISFYing $\mathcal{K}^{*}(\xi, U) \cdot R^{*}=0$}

Theorem 8.1. If in an $n$-dimensional $(n>2)$ LP-Sasakian manifold $M$, the condition $\mathcal{K}^{*}(\xi, U) \circ R^{*}=0$ holds, then the equation $S^{2}(Y, U)+9 \psi^{2} g(Y, U)+$ $\left[(n-1)^{2}+9 \psi^{2}\right] \eta(Y) \eta(U)+6 \psi S(Y, \phi U)=0$, is satisfied on $M$, where $Y, U$ $\in \chi(M)$ and $\psi=\operatorname{trace}(\phi)$.

Proof. Let us consider an LP-Sasakian manifold $M$ satisfying the condition

$$
\left(\mathcal{K}^{*}(\xi, U) \cdot R^{*}\right)(X, Y) Z=0 .
$$

Then, we have

$$
\begin{aligned}
0= & \mathcal{K}^{*}(\xi, U) R^{*}(X, Y) Z-R^{*}\left(\mathcal{K}^{*}(\xi, U) X, Y\right) Z \\
& -R^{*}\left(X, \mathcal{K}^{*}(\xi, U) Y\right) Z-R^{*}(X, Y) \mathcal{K}^{*}(\xi, U) Z .
\end{aligned}
$$

Replacing $Z$ by $\xi$ in (77), we get

$$
\begin{aligned}
0= & \mathcal{K}^{*}(\xi, U) R^{*}(X, Y) \xi-R^{*}\left(\mathcal{K}^{*}(\xi, U) X, Y\right) \xi \\
& -R^{*}\left(X, \mathcal{K}^{*}(\xi, U) Y\right) \xi-R^{*}(X, Y) \mathcal{K}^{*}(\xi, U) \xi .
\end{aligned}
$$

In view of (37), (40), (3) and (78), we have

$$
\begin{aligned}
0 & =R^{*}(X, Y) \mathcal{K}^{*}(\xi, U) \xi \\
& =R^{*}(X, Y) Q^{*} U \\
& =R^{*}(X, Y) Q U+3 \psi R^{*}(X, Y) \phi U .
\end{aligned}
$$

The inner product of the equation (79) with vector field $V$ gives

$$
0=g\left(R^{*}(X, Y) Q U, V\right)+3 \psi g\left(R^{*}(X, Y) \phi U, V\right) .
$$


Let $\left\{e_{i}\right\}(1 \leq i \leq n)$ be an orthonormal basis of the tangent space at any point of the manifold $M$. Setting $X=V=e_{i}$ and taking summation over $i(1 \leq i \leq n)$ and using (18) in (80), we get

$$
\begin{aligned}
0= & S^{2}(Y, U)+9 \psi^{2} g(Y, U) \\
& +\left[(n-1)^{2}+9 \psi^{2}\right] \eta(Y) \eta(U)+6 \psi S(Y, \phi U) .
\end{aligned}
$$

This gives the theorem.

\section{ACKnowledgement}

The authors would like to thank the referee for their valuable suggestions to improve the paper.

\section{REFERENCES}

[1] Biswas, A. and Baishya, K. K., "Study on generalized pseudo (Ricci) symmetric Sasakian manifold admitting general connection", Bulletin of the Transilvania University of Brasov, 12(2) (2020), 233-246.

[2] Biswas, A. and Baishya, K. K., "A general connection on Sasakian manifolds and the case of almost pseudo symmetric Sasakian manifolds", Scientific Studies and Research Series Mathematics and Informatics, 29(1) (2019), 59-72.

[3] Blaga, A. M., "Canonical connection on Para Kenmotso manifold", Novi Sad J. Math., 45(2) (2015), 131-142.

[4] Blair, D. E., "Contact manifolds in Riemannian Geometry", Lect. Notes Math. SpringerVerlag, Berlin, 509 (1976).

[5] Das, A. and Mandal, A., "Study of Ricci solitons on concircularly flat Sasakian manifolds admitting Zamkovoy connection", The Aligarh Bull. of Math., 39(2) (2020), 47-61.

[6] De, U. C. and Guha, N., On generalized recurrent manifolds, J. Nat. Acad. Math., 9 (1991), 85-92.

[7] De, U. C., Matsumoto, K. and Shaikh, A. A., "On Lorentzian para-Sasakian manifolds", Rendiconti del Seminario Matematico di Messina, Serie II, Supplemento, 3 (1999), 149-158.

[8] Dubey, R. S., "Generalized recurrent spaces", Indian j. pure Appl. Math., 10(12) (1979), 1508-1513.

[9] Ishii, Y., "On conharmonic transformations", Tensor, N. S., 11 (1957), 73-80.

[10] Mandal, A. and Das, A., "On M-Projective Curvature Tensor of Sasakian Manifolds admitting Zamkovoy Connection", Adv. Math. Sci. J., 9(10) (2020), 8929-8940.

[11] Mandal, A. and Das, A., "Projective Curvature Tensor with respect to Zamkovoy connection in Lorentzian para Sasakian manifolds", J. Indones. Math. Soc., 26(3) (2020), 369-379.

[12] Mandal, A. and Das, A., "Pseudo projective curvature tensor on Sasakian manifolds admitting Zamkovoy connection", Bull. Cal. Math. Soc., 112(5) (2020), 431-450.

[13] Matsumoto, K., "On Lorentzian paracontact manifolds", Bull. of Yamagata Univ. Nat. Sci., 12(2) (1989), 151-156.

[14] Mihai, I. and Rosca, R., "On Lorentzian P-Sasakian manifolds, Classical Analysis", World Scientific Publi., (1992), 155-169.

[15] Shaikh, A. A., Prakasha, D. G. and Ahmad, H., "On generalized $\phi$-recurrent LP-Sasakian manifolds", J. of the Egyptian Mathematical Society, 23 (2015), 161-166.

[16] Taleshian, A., Prakasha, D. G., Vikas, K. and Asghari, N., "On The conharmonic Curvature Tensor of LP-Sasakian Manifolds", Palestine J. of Math., 5(1) (2016), 177-184.

[17] Ozgur, C., " $\varphi$-Conformally flat Lorentzian para Saskian manifolds", Radovi Mathemeticki, 12 (2003), 99-106.

[18] Zamkovoy, S., "Canonical connections on paracontact manifolds", Ann. Global Anal. Geom., 36(1) (2008), 37-60. 